\title{
OPEN Predicting the probability of Gaucher disease in subjects with splenomegaly and thrombocytopenia
}

\begin{abstract}
Irene Motta ${ }^{1,2}$, Dario Consonni ${ }^{3}$, Marina Stroppiano $^{4}$, Christian Benedetto $^{5}$, Elena Cassinerio ${ }^{1}$, Barbara Tappino ${ }^{4}$, Paola Ranalli ${ }^{6}$, Lorenza Borin ${ }^{7}$, Luca Facchini ${ }^{8}$, Andrea Patriarca9 Wilma Barcellini ${ }^{10}$, Federica Lanza ${ }^{4}$, Mirella Filocamo ${ }^{4}$, Maria Domenica Cappellini ${ }^{1,2}{ }^{\circledR}$ \& Splenomegaly Gaucher group*

Hematologists are frequently involved in the diagnostic pathway of Gaucher disease type 1 (GD1) patients since they present several hematological signs. However, GD1 is mainly underdiagnosed because of a lack of awareness. In this multicenter study, we combine the use of a diagnostic algorithm with a simple test ( $\beta$-glucosidase activity on Dried Blood Spot) in order to facilitate the diagnosis in a population presenting to the hematologist with splenomegaly and/or thrombocytopenia associated with other hematological signs. In this high-risk population, the prevalence of GD1 is 3.3\%. We propose an equation that predicts the probability of having GD1 according to three parameters that are routinely evaluated: platelet count, ferritin, and transferrin saturation.
\end{abstract}

Gaucher disease type 1 (GD1) is an autosomal recessive lysosomal storage disorder caused by mutations in the GBA gene resulting in the deficiency of $\beta$-glucosidase enzyme. Its prevalence in the non-Ashkenazy Jewish population is estimated at 1:40,000-100,000 subjects, whereas in Ashkenazi Jewish is 1:500-1000.

At diagnosis, patients present with several hematological signs and symptoms, including splenomegaly (86\%), anemia (64\%), thrombocytopenia (56\%), bleeding history, and monoclonal gammopathy of undetermined significance (MGUS), leading them to consult a hematologist on their diagnostic pathway ${ }^{1}$. However, an international survey showed that only $20 \%$ of hematologists include GD1 in the differential diagnosis of a patient with anemia, thrombocytopenia, hepatomegaly, splenomegaly, and bone pain ${ }^{2}$. As a matter of fact, GD1 is misdiagnosed and underdiagnosed; thus, patients often experience long diagnostic delays, leading to inappropriate procedures, treatments, and complications that often cannot be reversed by the available treatments ${ }^{2}$.

Moreover, half of the patients are diagnosed through bone marrow biopsy, although the diagnostic gold standard is the activity of $\beta$-glucosidase on leucocytes or fibroblasts ${ }^{3}$. Among the crucial obstacles to diagnosis, physicians mainly identify outsourced testing and, more importantly, the lack of awareness ${ }^{4}$. Thus, ten years ago, a panel of experts published two diagnostic algorithms, one for the Ashkenazi and one for the non-Ashkenazi Jewish population, to facilitate the diagnosis of GD1 for hematologists ${ }^{5}$.

The new-born screening has been experimented in some areas, showing an incidence of 1:22,205 in Northern Italy ${ }^{6}$. However, the large-scale implementation of new-born screening for a disease with high phenotypic heterogeneity, ranging from asymptomatic to severely symptomatic conditions, should be carefully evaluated.

We hypothesized that an approach that combines a diagnostic algorithm and a simple, cheap, and easy-todo test could facilitate the diagnosis. We designed a multicenter study that aimed at evaluating the prevalence

\footnotetext{
${ }^{1}$ General Medicine Unit, Rare Diseases Center, Fondazione IRCCS Ca' Granda Ospedale Maggiore Policlinico, Via F. Sforza, 35, 20122 Milan, Italy. ${ }^{2}$ Department of Clinical Sciences and Community Health, Università Degli Studi Di Milano, Milan, Italy. ${ }^{3}$ Epidemiology Unit, Fondazione IRCCS Ca' Granda Ospedale Maggiore Policlinico, Milan, Italy. ${ }^{4}$ Laboratorio Di Genetica Molecolare E Biobanche, Istituto G. Gaslini, Genoa, Italy. ${ }^{5}$ Università Degli Studi Di Milano, Milan, Italy. ${ }^{6} \mathrm{Hemophilia}$ and Rare Blood Diseases Centre, Oncology and Hematology Department, S. Spirito Hospital, Pescara, Italy. ${ }^{7}$ Hematology Division, San Gerardo Hospital, Monza, Italy. ${ }^{8}$ Division of Hematology, Azienda USL-IRCCS Di Reggio Emilia, Reggio Emilia, Italy. ${ }^{9}$ Division of Hematology, Department of Translational Medicine, University of Eastern Piedmont and Ospedale Maggiore Della Carità, Novara, Italy. ${ }^{10} \mathrm{Hematology}$ Unit, Fondazione IRCCS Ca' Granda Ospedale Maggiore Policlinico, Milan, Italy. ${ }^{*} \mathrm{~A}$ list of authors and their affiliations appears at the end of the paper. ${ }^{\bowtie}$ email: maria.cappellini@unimi.it
} 
of GD1 in a high-risk population presenting to the hematologist with splenomegaly and/or thrombocytopenia associated with other hematological signs or symptoms suggestive of GD1. Preliminary results of this study on the first 196 patients have been previously published, showing a GD1 prevalence of 3.6\% in a high-risk population ${ }^{7}$.

\section{Materials and methods}

Study design. We designed a multicenter study among 35 hematology centers in Italy. According to the feasibility questionnaire, we expected to enroll 500 subjects. The enrolment started in September 2010 and closed in December 2018.

Inclusion and exclusion criteria were based on the published algorithm for the non-Ashkenazi population ${ }^{5}$ :

- Inclusion criteria: splenomegaly and/or thrombocytopenia and at least one sign or symptom among bone pain history, anemia, MGUS, polyclonal gammopathy in subjects under 30 years of age, splenectomy;

- Exclusion criteria: onco-hematological diseases, portal hypertension due to liver diseases, hemoglobinopathies, or chronic hemolytic anemias.

Demographic, clinical, and laboratory data were collected at enrolment, gathered in a specific case report form, and collected by the coordinating center at Fondazione IRCCS Ca' Granda Ospedale Maggiore Policlinico, Milan, Italy.

Beta-glucosidase activity. The beta-glucosidase activity on Dried Blood Spot (DBS) was centralized at Ospedale Gaslini, Genoa, Italy ${ }^{8,9}$. Normal values range from 4.4 to $17.7 \mathrm{pmol} / \mathrm{punch} / \mathrm{h}$. Subjects showing activity below $4.4 \mathrm{pmol} / \mathrm{punch} / \mathrm{h}$ were recalled to be assessed with the gold standard assay on nucleated cell homogenates (leucocytes, EBV-lymphoblasts, or fibroblasts). If the enzymatic defect was confirmed, the diagnosis was completed with the molecular $G B A$ analysis.

Statistical analysis. The prevalence of GD1 and its $95 \%$ confidence interval (CI) based on the exact method were calculated. Demographic, clinical, and laboratory variables of patients affected by GD1 were compared to those of unaffected patients using Wilcoxon rank-sum (Mann-Whitney U-test) test (for continuous variables) or Fisher's exact test (for categorical variables). Using univariate and multiple logistic regression models, we analyzed the predictive role of platelets (thousands $/ \mathrm{mm}^{3}$ ), ferritin $(\mu \mathrm{g} / \mathrm{L})$, transferrin saturation (TSAT) $(\%)$ in this high-risk population. Furthermore, we considered the three variables jointly, and we calculated the respective areas under the curve (AUC) of the receiver operating characteristic (ROC) curves. Analyses were performed with Stata 16 (StataCorp. 2019. Stata: Release 16. Statistical Software; StataCorp LP, College Station, TX, USA).

Ethical aspects. The study was approved by the ethical review committee of the coordinating center "Comitato Etico Milano Area 2" (Protocol number 714/10) and by all participating Centers, and was carried out in compliance with the principles established in the Helsinki Declaration. Informed consent was obtained from all individual participants included in the study.

\section{Results}

The prevalence of GD1 in high risk predominantly Caucasian population is above $3 \%$. Five hundred subjects have been enrolled in the study. Forty-five have been excluded because they did not fulfill the inclusion and exclusion criteria (Fig. 1). Demographic, clinical, and laboratory characteristics are presented in Table 1. Ninety-one percent (91\%) of the subjects were Caucasian. The mean age at enrolment was 46.9 years, and $31.9 \%(145 / 455)$ were females. The majority had splenomegaly $(89.7 \%)$, and approximately half $(47.9 \%)$ thrombocytopenia associated with other signs/symptoms. Anemia was the most common adjunctive sign (23.1\%) (Table 1).

DBS showed normal values in 379 subjects, while 76 (16.7\%) had a reduced $\beta$-glucosidase activity. These 76 patients and a patient with a family history of GD1 presenting with a $\beta$-glucosidase activity slightly above the lower normal range were recalled to test the conventional enzymatic activity. Among the 65 patients tested with $\beta$-glucosidase activity on nucleated cell homogenates (12 did not answer), 15 were diagnosed with Gaucher disease type 1 , with a prevalence of $3.3 \%(15 / 455,95 \%$ CI 1.9-5.4\%). In 14, the molecular analysis of the GBA gene identified the mutations. In one patient, no mutations of GBA gene nor PSAP, encoding for saposin C, were identified. Among GD1 patients, 7/15 (46.7\%) were female, 14 of Caucasian origin, and the mean age at diagnosis was 43.5 years. They showed a lower platelet count compared to non-GD1 patients $\left(84.000 / \mathrm{mm}^{3} \mathrm{vs} 131.000 /\right.$ $\left.\mathrm{mm}^{3}, p=0.0006\right)$, a higher serum ferritin level $(551 \mathrm{ng} / \mathrm{dL}$ vs $139 \mathrm{ng} / \mathrm{dL}, p=0.0002)$ which was associated to a lower transferrin saturation $(20.8 \%$ vs $25.7 \%, p=0.03)$.

Platelet count, ferritin, and transferrin saturation predict the probability of having GD1 in this high-risk population. Considering 159 subjects (13 with GD1) with complete data on ferritin, platelets, and TSAT, the best discrimination between GD1 and non-GD1 subjects was provided by platelets (AUC $=0.79$ ), while ferritin and transferrin saturation showed lower AUCs (Fig. 2).

The joint analysis of these three variables together in a multiple logistic regression model yielded an AUC of 0.89 (95\% CI: 0.82-0.96). The corresponding equation is: 


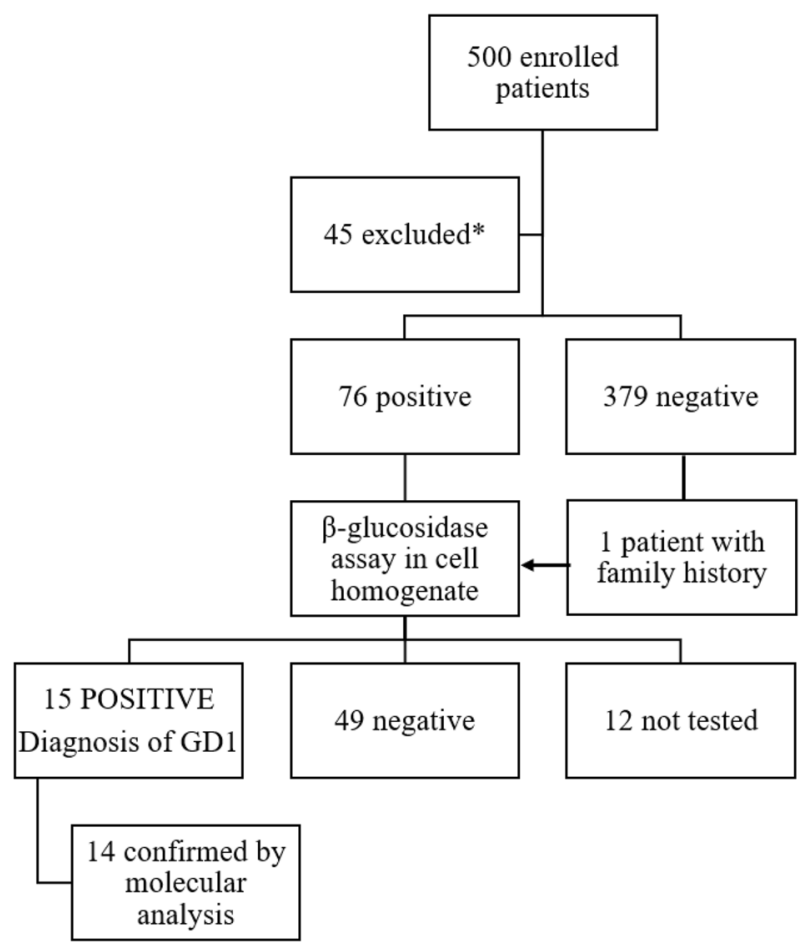

Figure 1. Results of DBS, $\beta$-glucosidase assay in cell homogenate, and molecular analysis of the enrolled patients. DBS dried blood spot, GD1 Gaucher disease type $1 .{ }^{\star}$ They did not fulfill the inclusion and exclusion criteria.

$$
\operatorname{odds}(\mathrm{GD} 1)=\exp [0.311+(0.002 \times \mathrm{F})+(-0.018 \times \mathrm{P})+(-0.069 \times \mathrm{TS})]
$$

where $\mathrm{F}=$ ferritin $(\mu \mathrm{g} / \mathrm{L}), \mathrm{P}=$ platelets (thousands $\left./ \mathrm{mm}^{3}\right)$, and $\mathrm{TS}=$ transferrin saturation $(\%)$.

Hence the predicted probability of GD1 in patients presenting with splenomegaly and/or thrombocytopenia plus the abovementioned ancillary signs can be calculated as: $100 \times[\operatorname{odds}(\mathrm{GD} 1) /[1+$ odds(GD1)].]

When the probability predicted by the equation was $<5 \%$, we observed only one subject with GD1 out of 93 (1.1\%). When the predicted probability was 5 to $<10 \%$, the observed frequency was $3.9 \%(1 / 26)$. With predicted probabilities $\geq 10 \%$, the observed GD1 prevalence was substantially higher $(11 / 30=27.5 \%)$.

The addition of a history of bone pain to this model did not lead to an AUC increase, and thus, this variable was omitted.

\section{Discussion}

This study shows that in a predominantly Caucasian high-risk population presenting to the hematologist with splenomegaly and/or thrombocytopenia associated with other hematological signs, including anemia and MGUS, $3.3 \%$ of patients have Gaucher disease. These data confirm our previously published preliminary data ${ }^{7}$. Similar studies have been reproduced in different regions worldwide, with different prevalence, ranging from no cases detected in a Canadian study on 221 subjects $^{10}$ to $7.0 \%$ in an adult cohort in China ${ }^{11}$. A similar approach is under evaluation in the Italian pediatric population ${ }^{12}$, and promising preliminary results have been presented in a Chinese study ${ }^{13}$. Altogether, these data support the use of the previously published algorithm by Mistry et al. ${ }^{5}$ associated with a simple first-level diagnostic test to screen high-risk populations. Diagnostic confirmation with $\beta$-glucosidase activity on nucleated cell homogenates is necessary to confirm the diagnosis. Of note, given that false negatives in the DBS test may arise due to methodological differences in blood spot drying, transport and storage $^{14-16}$ testing with the gold standard diagnostic exam is warranted when there is any clinical suspicion of Gaucher disease, even in the presence of normal DBS values.

Since lysosomal storage disorders, including GD1, are underdiagnosed, other approaches have been proposed to increase the diagnostic rate. Namely, the new-born screening has been experimented in several regions with different results according to the ethnicity of the tested population ${ }^{6,17-19}$. However, the new-born screening raises unique issues that are primarily related to the inevitable detection of a disease with late-onset phenotypes.

Among the enrolled subjects, the only clearly different parameters between GD1 and non-GD1 patients were platelet count and serum ferritin. Hyperferritinemia with normal transferrin saturation is a common finding in naïve patients with $\mathrm{GD}^{20}$, with prevalence ranging between 63 and $81 \%^{21,22}$. Recently, together with other Italian 


\begin{tabular}{|c|c|c|c|}
\hline Variable & Non-GD1 patients $(n=440)$ & GD1 patients $(n=15)$ & $p$ value \\
\hline Sex, F & $138(31.3 \%)$ & $7(46.7 \%)$ & 0.26 \\
\hline Ethnicity $\mathrm{C} / \mathrm{J} / \mathrm{O}$ & $400 / 2 / 38$ & $14 / 0 / 1$ & 0.28 \\
\hline Age, mean \pm SD & $47.0 \pm 17.5$ & $43.5 \pm 15.1$ & 0.38 \\
\hline \multicolumn{4}{|l|}{ Inclusion criteria, n (\%) } \\
\hline Splenomegaly & $390(88.6 \%)$ & $14(93.3 \%)$ & 1.00 \\
\hline Splenomegaly alone & $205(46.6 \%)$ & $4(26.7 \%)$ & 0.19 \\
\hline Thrombocytopenia & $208(47.3 \%)$ & $10(66.7 \%)$ & 0.11 \\
\hline Thrombocytopenia alone & $35(7.9 \%)$ & $1(6.7 \%)$ & 1.00 \\
\hline Anemia & $101(23.0 \%)$ & $4(26.7 \%)$ & 0.75 \\
\hline Bone pain & $67(15.2 \%)$ & $6(40.0 \%)$ & 0.02 \\
\hline MGUS & $26(6.0 \%)$ & $2(13.3 \%)$ & 0.23 \\
\hline Polyclonal gammopathy U30 & $4(0.9 \%)$ & 0 & 1.00 \\
\hline Splenectomy & $8(1.8 \%)$ & 0 & 1.00 \\
\hline \multicolumn{4}{|l|}{ History of } \\
\hline Bone pain & $76(17.8 \%)$ & $6(46.2 \%)$ & 0.02 \\
\hline Fractures & $2(0.5 \%)$ & 0 & 1.00 \\
\hline Bleeding & $22(5.2 \%)$ & $1(7.1 \%)$ & 0.53 \\
\hline Gallstones & $52(12.4 \%)$ & $4(26.7 \%)$ & 0.11 \\
\hline Growth retardation & $5(1.2 \%)$ & $1(7.1 \%)$ & 0.38 \\
\hline \multicolumn{4}{|l|}{ Laboratory parameters } \\
\hline $\mathrm{Hb}$, mean $\pm \mathrm{SD}(\mathrm{g} / \mathrm{dL})$ & $13.3 \pm 2.4$ & $12.9 \pm 2.1$ & 0.28 \\
\hline $\mathrm{WBC}$, mean $\pm \mathrm{SD}\left(\times 10^{3} / \mathrm{mm}^{3}\right)$ & $5.6 \pm 2.3$ & $4.5 \pm 1.6$ & 0.048 \\
\hline $\mathrm{RBC}$, mean $\pm \mathrm{SD}\left(\times 10^{6} / \mathrm{mm}^{3}\right)$ & $4.8 \pm 0.8$ & $4.6 \pm 0.6$ & 0.20 \\
\hline PLT, median/range $\left(\times 10^{3} / \mathrm{mm}^{3}\right)$ & $131 / 8-767$ & $84 / 44-148$ & 0.0006 \\
\hline Iron, mean $\pm S D(\mu \mathrm{g} / \mathrm{dL})$ & $87.3 \pm 44.7$ & $76.5 \pm 30.0$ & 0.52 \\
\hline Ferritin, median/range $(\mu \mathrm{g} / \mathrm{L})$ & $139 / 6-2858$ & $551 / 55-1547$ & 0.0002 \\
\hline Transferrin, mean \pm SD $(\mathrm{mg} / \mathrm{dL})$ & $234 \pm 50$ & $269 \pm 51$ & 0.03 \\
\hline TSAT, median/range (\%) & $25.7 / 5.9-87.2$ & $20.8 / 7.2-26.7$ & 0.03 \\
\hline ALT, median/range (UI/L) & $29.8 / 1-496$ & $25.7 / 1-57$ & 0.98 \\
\hline AST, median/range (UI/L) & $28.4 / 1-685$ & $29.3 / 17-51$ & 0.19 \\
\hline ALP, median/range (UI/L) & $78.5 / 4-627$ & $74.5 / 39-100$ & 0.44 \\
\hline Cholesterol, mean $\pm \mathrm{SD}(\mathrm{mg} / \mathrm{dL})$ & $157.7 \pm 52.2$ & $150.2 \pm 31.8$ & 0.84 \\
\hline \multicolumn{4}{|l|}{ Serum protein electrophoresis } \\
\hline MGUS & $17(3.9 \%)$ & $2(13.3 \%)$ & 0.12 \\
\hline Bipolar spleen diameter, mean \pm SD $(\mathrm{cm})$ & $15.5 \pm 2.4$ & $17.1 \pm 4.2$ & 0.33 \\
\hline
\end{tabular}

Table 1. Demographic, clinical, and laboratory characteristics. GD1: Gaucher disease type 1; C: Caucasian; J: Jewish; O: other; MGUS: monoclonal gammopathy of unknown significance; U30: under 30 years of age; Hb: hemoglobin: WBC: white blood count: RBC: red blood cells; PLT platelets; TSAT: transferrin saturation; ALT: alanine aminotransferase; AST: aspartate aminotransferase; ALP: alkaline phosphatase.

referral groups for iron disorders, we have proposed a new diagnostic flow-chart ${ }^{23}$, which enhances hyperferritinemia role when associated with splenomegaly and thrombocytopenia.

Here we propose an equation that predicts the probability of having GD1 according to platelet count and ferritin and TSAT levels and thus may support hematologists when evaluating a subject with splenomegaly and/ or thrombocytopenia.

\section{Conclusion}

High-risk population testing is effective in identifying Gaucher disease patients who present to the hematologist with splenomegaly and/or thrombocytopenia. The evaluation of the probability of having GD1 according to an equation and the use of DBS as a first-level test are potentially useful tools that can facilitate the diagnostic process. 


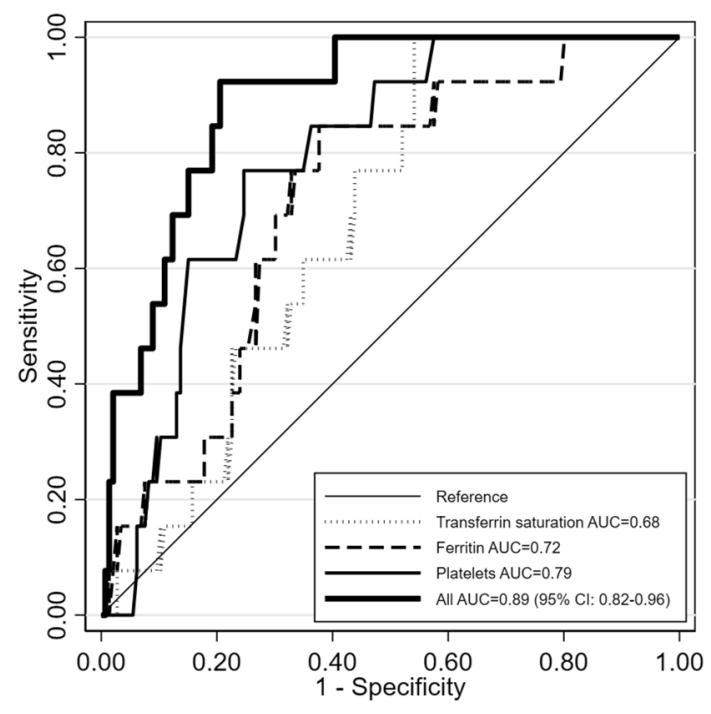

Figure 2. Receiver operating characteristic (ROC) curves of GD1 for platelets (thousands/ $\mathrm{mm}^{3}$ ), ferritin $(\mu \mathrm{g} / \mathrm{L}$ ), transferrin saturation (\%), and for the three variables jointly analyzed in a multiple logistic regression model. $A U C$ area under the curve, $C I$ confidence interval.

Received: 26 October 2020; Accepted: 14 January 2021

Published online: 28 January 2021

\section{References}

1. Charrow, J. et al. The Gaucher registry: Demographics and disease characteristics of 1698 patients with Gaucher disease. Arch. Intern. Med. 160, 2835-2843. https://doi.org/10.1001/archinte.160.18.2835 (2000).

2. Mistry, P. K., Sadan, S., Yang, R., Yee, J. \& Yang, M. Consequences of diagnostic delays in type 1 Gaucher disease: The need for greater awareness among hematologists-oncologists and an opportunity for early diagnosis and intervention. Am. J. Hematol. 82, 697-701. https://doi.org/10.1002/ajh.20908 (2007).

3. Thomas, A. S., Mehta, A. B. \& Hughes, D. A. Diagnosing Gaucher disease: An on-going need for increased awareness amongst haematologists. Blood Cells Mol. Dis. 50, 212-217. https://doi.org/10.1016/j.bcmd.2012.11.004 (2013).

4. Mehta, A. et al. Exploring the patient journey to diagnosis of Gaucher disease from the perspective of 212 patients with Gaucher disease and 16 Gaucher expert physicians. Mol. Genet. Metab. 122, 122-129. https://doi.org/10.1016/j.ymgme.2017.08.002 (2017).

5. Mistry, P. K. et al. A reappraisal of Gaucher disease-diagnosis and disease management algorithms. Am. J. Hematol. 86, 110-115. https://doi.org/10.1002/ajh.21888 (2011).

6. Burlina, A. B. et al. Newborn screening for lysosomal storage disorders by tandem mass spectrometry in North East Italy. J. Inherit. Metab. Dis. 41, 209-219. https://doi.org/10.1007/s10545-017-0098-3 (2018).

7. Motta, I. et al. A multicentre observational study for early diagnosis of Gaucher disease in patients with Splenomegaly and/or Thrombocytopenia. Eur. J. Haematol. 96, 352-359. https://doi.org/10.1111/ejh.12596 (2016).

8. Stroppiano, M. et al. Validity of beta-D-glucosidase activity measured in dried blood samples for detection of potential Gaucher disease patients. Clin. Biochem. 47, 1293-1296. https://doi.org/10.1016/j.clinbiochem.2014.06.005 (2014).

9. Olivova, P. et al. An improved high-throughput dried blood spot screening method for Gaucher disease. Clin. Chim. Acta 398, 163-164. https://doi.org/10.1016/j.cca.2008.08.024 (2008).

10. Russell, S. A. et al. Gaucher disease screening at a general adult hematology tertiary care centre: A prospective study. Int. J. Lab. Hematol. 41, e66-e69. https://doi.org/10.1111/ijlh.12960 (2019).

11. Huang, Y. et al. High risk screening for Gaucher disease in patients with splenomegaly and/or thrombocytopenia in China: 55 cases identified. Clin. Chim. Acta 506, 22-27. https://doi.org/10.1016/j.cca.2020.03.016 (2020)

12. Di Rocco, M. et al. Early diagnosis of Gaucher disease in pediatric patients: Proposal for a diagnostic algorithm. Pediatr. Blood Cancer 61, 1905-1909. https://doi.org/10.1002/pbc.25165 (2014).

13. Lei, K. et al. A pilot screening of high-risk Gaucher disease children using dried blood spot methods in Shandong province of China. Orphanet J. Rare Dis. 13, 48. https://doi.org/10.1186/s13023-018-0782-x (2018).

14. Elbin, C. S. et al. The effect of preparation, storage and shipping of dried blood spots on the activity of five lysosomal enzymes. Clin. Chim. Acta 412, 1207-1212. https://doi.org/10.1016/j.cca.2011.03.012 (2011).

15. Supriya, M., De, T. \& Christopher, R. Effect of temperature on lysosomal enzyme activity during preparation and storage of dried blood spots. J. Clin. Lab. Anal. 32(1), e22220. https://doi.org/10.1002/jcla.22220 (2018).

16. Mei, J. V., Alexander, J. R., Adam, B. W. \& Hannon, W. H. Use of filter paper for the collection and analysis of human whole blood specimens. J. Nutr. 131, 1631S-1636S. https://doi.org/10.1093/jn/131.5.1631S (2001).

17. Burton, B. K. et al. Newborn screening for lysosomal storage disorders in Illinois: The initial 15-month experience. J. Pediatr. 190, 130-135. https://doi.org/10.1016/j.jpeds.2017.06.048 (2017).

18. Hopkins, P. V. et al. Lysosomal storage disorder screening implementation: Findings from the first six months of full population pilot testing in Missouri. J. Pediatr. 166, 172-177. https://doi.org/10.1016/j.jpeds.2014.09.023 (2015).

19. Wasserstein, M. P. et al. The New York pilot newborn screening program for lysosomal storage diseases: Report of the first 65,000 infants. Genet. Med. 21, 631-640. https://doi.org/10.1038/s41436-018-0129-y (2019).

20. Stein, P., Yu, H., Jain, D. \& Mistry, P. K. Hyperferritinemia and iron overload in type 1 Gaucher disease. Am. J. Hematol. 85, $472-476$. https://doi.org/10.1002/ajh.21721 (2010).

21. Lefebvre, T. et al. Involvement of hepcidin in iron metabolism dysregulation in Gaucher disease. Haematologica 103, 587-596. https://doi.org/10.3324/haematol.2017.177816 (2018). 
22. Lorenz, F. et al. Ferritinemia and serum inflammatory cytokines in Swedish adults with Gaucher disease type 1. Blood Cells Mol. Dis. 68, 35-42. https://doi.org/10.1016/j.bcmd.2016.10.010 (2018).

23. Marchi, G. et al. Hyperferritinemia and diagnosis of type 1 Gaucher disease. Am. J. Hematol. 95, 570-576. https://doi.org/10.1002/ ajh.25752 (2020).

\section{Author contributions}

M.D.C. designed the research study. I.M., E.C., P.R., L.B., L.F., A.P., W.B. enrolled the patients. M.S., M.F., B.T., F.L. performed the tests. I.M. and C.B. collected the data. D.C., I.M. and C.B. analyzed the data. I.M. and D.C. wrote the paper. All authors contributed to critical revision and final approval of the version to be published.

\section{Funding}

This work was supported by a grant from Sanofi Genzyme.

\section{Competing interests}

IM received Lecture Honoraria from Sanofi Genzyme and is a member of Sanofi Genzyme advisory board. WB is a member of Agios, Alexion, Apellis, Biocryst, Bioverativ, Incyte, Momenta, Novartis advisory board, received lecture honoraria from Alexion, Incyte, Novartis, Sanofi and research support from Alexion. MDC is a member of Vifor, Sanofi Genzyme, Celgene, Novartis, and Bluebird advisory board. DC, MS, CB, EC, BT, PR, LB, LF, AP, FL, MF have no conflict of interest.

\section{Additional information}

Correspondence and requests for materials should be addressed to M.D.C.

Reprints and permissions information is available at www.nature.com/reprints.

Publisher's note Springer Nature remains neutral with regard to jurisdictional claims in published maps and institutional affiliations.

(c) (i) Open Access This article is licensed under a Creative Commons Attribution 4.0 International License, which permits use, sharing, adaptation, distribution and reproduction in any medium or format, as long as you give appropriate credit to the original author(s) and the source, provide a link to the Creative Commons licence, and indicate if changes were made. The images or other third party material in this article are included in the article's Creative Commons licence, unless indicated otherwise in a credit line to the material. If material is not included in the article's Creative Commons licence and your intended use is not permitted by statutory regulation or exceeds the permitted use, you will need to obtain permission directly from the copyright holder. To view a copy of this licence, visit http://creativecommons.org/licenses/by/4.0/.

(C) The Author(s) 2021

\section{Splenomegaly Gaucher group}

\section{Francesca Farina ${ }^{7,12}$, Katia Codeluppi ${ }^{8}$, Elena Rivolti ${ }^{8}$, Federico Simonetti ${ }^{11}$, Francesca Lunghi $^{12}$, Tommasina Perrone ${ }^{13}$, Nicola Sgherza ${ }^{13}$, Valentina Carrai ${ }^{14}$, Anna Maria Cafro ${ }^{15}$, Roberto Cairoli ${ }^{15}$, Angela Amendola ${ }^{16}$, Elena Trabacchi ${ }^{17}$, Daniele Vallisa ${ }^{17}$, Ilaria Burgo ${ }^{18}$, Augusto Bramante Federici ${ }^{18}$, Cecilia Carbone ${ }^{19}$, Mariella D'Adda ${ }^{19}$, Donato Mannina ${ }^{20}$, Valeria Di Giacomo ${ }^{20}$, Giulia Lupparelli ${ }^{21}$ \& Alessandra Lombardo ${ }^{21}$}

${ }^{11}$ UOC Dipartimentale Ematologia, Azienda USL Nord-Ovest Toscana, Ospedale Versilia, Camaiore, Italy. ${ }^{12}$ Hematology and Bone Marrow Transplantation Unit, San Raffaele Scientific Institute, IRCCS Milano, Milan, Italy. ${ }^{13}$ University of Bari, Bari, Italy. ${ }^{14}$ SODc Ematologia Azienda Ospedaliero Universitaria Careggi, Firenze, Italy. ${ }^{15}$ Department of Hematology, ASST Grande Ospedale Metropolitano, Niguarda, Milan, Italy. ${ }^{16}$ San Carlo Hospital, Potenza, Italy. ${ }^{17} \mathrm{Hematology}$, Piacenza, Italy. ${ }^{18} \mathrm{Hematology}$ and Transfusion Medicine, L Sacco University Hospital, Milan, Italy. ${ }^{19}$ Hematology Department, ASST Spedali Civili, Brescia, Italy. ${ }^{20}$ Division of Hematology, Azienda Ospedaliera Papardo, Messina, Italy. ${ }^{21}$ Hematology Unit, Azienda Ospedaliera Santa Maria di Terni, University of Perugia, Terni, Italy. 